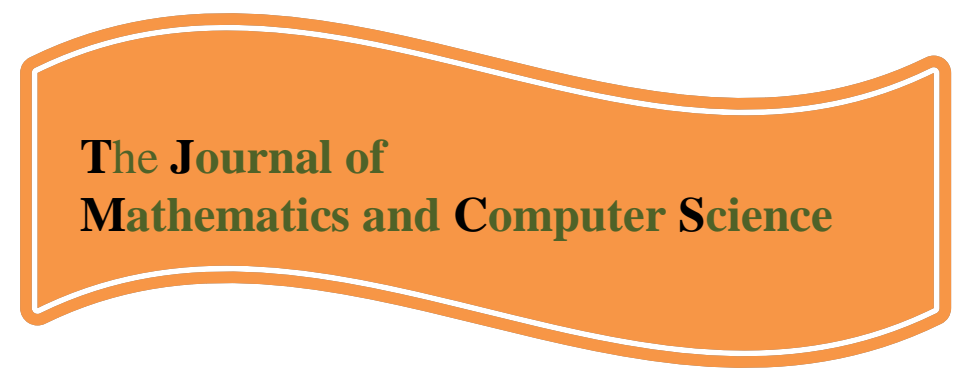

Available online at

http://www.TIMCS.com

The Journal of Mathematics and Computer Science Vol .4 No.4 (2012) 570-584

\title{
Investigation Of The Effect Of Earthquake On Concrete Minaret Under Static Loads Using Genetic programing
}

\author{
Kaveh Kumarci \\ Sama technical and vocational training college, Islamic Azad University, Shahrekord Branch, \\ Shahr-e-kord, Iran \\ email: kumarci kaveh@yahoo.com \\ Afsaneh Banitalebi Dehkordi \\ 2Department Of Computer Science,Payam Noor University, PO BOX 19395-3697 Tehran, I.R Iran \\ email: banitalebi2012@yahool.com
}

Received: October 2012, Revised: November 2012

Online Publication: November 2012

\begin{abstract}
Minarets are special structures commonly used in Islamic architectures. The seismic behaviors of minarets is quite different from that the other well known structures because of their unique structural characteristics such as slenderness, distinctive geometrical shape and support system. Post earthquake observations indicate that there is a direct relationship between site selection and overall minaret behavior and damage. This study investigates the seismic response of cylindrical concrete minarets with circular cross section under static loads using genetic programing. Using SAP 2000 software, considered minarets were analyzed. At the first phase of study, according to regulation of designing of structures against earthquake (regulation No.2800), minarets dynamic responses were determined by a hand-operated analysis. Seismic analysis were carried out considering the design spectra defined by Iran structure design codes in Naqan, Shahr-e-kord. On the base of hand-operated and SAP2000 calculations the shear base and maximum lateral displacements were estimated.
\end{abstract}

Key words: Minaret, genetic programing, static loads, modeling, SAP2000 software.

\section{Introduction.}

A minaret is a slender tower built next to the mosque [12] which is used by Muezzin to call out the adhan in order to make people to come to prayers in Islam. Also it is used as a guide tower near the roads, mosques and caravanserai. 
A minaret basically consist of three parts: a base, a shaft and a gallery. The base is foundation of a minaret. The shaft is the thin, slim body of the minaret and stairs are place cylindrically in the shaft to provide the necessary structural support for the elongated shaft. The gallery is a balcony that encircles upper section where the muezzins call out to prayers [1].

A large number of research studies investigating the seismic response of historical minarets and towers are available $[3,4,8,13]$, but the most important structural properties should be known prior to analysis of structure [2].

One of the main goal of this research is study of concrete minarets, the response of them to earthquake and providing a suitable analysis method on the base of this structure specifications. In analysis of minaret in different modes, equal static analysis method is used. But some of these methods and obtaining exact solution are difficult and time consuming [7]. Also, optimum dimensions of minarets in the field of diameter, thickness and height were studied on the base of base shear and moment in minaret shell using SAP2000 software.

Providing a program to answer to multivariate problem as input or output is hard or impossible because we cant consider all of variables and their effect on each other [14]. Therefore getting to know Genetic programing and use of software can help to answer this problem.

Since genetic programing was born, it has been seen by some researchers in and out of the field that GP is a potentially powerful method for automated synthesis of computer programs by evolutionary means [11]. Genetic programing is a research method, belong to evaluation computation family and is a powerful method for automatically generating computer programs via the process of natural selection [6]. Genetic programing is the application of genetic algorithms [5,9] to creation of program code. It uses a genetic algorithm to search though a space of possible computer programs for one which is nearly optimal in its ability to perform a particular task [10]. Compared with Genetic Algorithm, GP can optimize more complex structures, so it use in various problems. At the second phases, a set of programs were generated. The better programs were selected from this set. GP model is used for some of minaret parameters such as diameter, thickness and height. Using genetic operators such as generation, mutation and cross over, we will get final population which are base shear and maximum lateral displacement in concrete minaret. Then, produced minaret samples will analyze using GP to find the best models for simulation of minaret behavior. Using GP and considering different diameter, thickness and height values (100 samples) in SAP2000, special models were provide for each minaret. Then, they compared with other 100 analyzed samples by SAP2000 and error percent was determined for each model.

\section{Static analysis of concrete minarets}

To study of static behavior of concrete minaret, height, diameter and thickness parameters were used which are 20-70 m, 2-7 m and 0.2-0.45 m, respectively. Also, considering three modes, computer models were provided:

1- Minaret with fixed diameter and height, but variable thickness

2- Minaret with fixed diameter and thickness, but variable height

3- Minaret with fixed height and thickness, but variable diameter

Static analysis of these three modes with shear base and maximum lateral displacement output are shown in figures 3 to 8 . Modeling hypothesis is as follow:

1- Minaret is build in Shahr-e-kord, Iran.

2- Minaret is made of armed concrete and $f^{\prime} c=210 \mathrm{~kg} / \mathrm{cm}^{2}, f y=4000 \mathrm{~kg} / \mathrm{cm}^{2}$, $v=0.2, E=2.188 \times 10^{9} \mathrm{~kg} / \mathrm{cm}^{2}$ and $\gamma_{c}=2400 \mathrm{~kg} / \mathrm{cm}^{3}$.

3- Minaret loading includes deed, seismic, thermal and earthquake loads in $\mathrm{X}$ and $\mathrm{Y}$ directions. For example, table 1 includes the results of hand-made and computer calculation in a cylinder minaret in which height, diameter and thickness are $30 \mathrm{~m}, 2 \mathrm{~m}$ 
and $20 \mathrm{~cm}$, respectively. In hand-made method, code of Iranian structure No.2800 and in computer method, SAP2000 software is used. In this table, A,I,R,T,B, C, W, V, K and U are design baseline acceleration, importance coefficient, behavior coefficient, period time, reflection coefficient, $\mathrm{ABI} / \mathrm{R}$, weight, base shear, rigidity and top displacement, respectively.

\begin{tabular}{|l|l|l|l|l|l|l|l|l|l|l|}
\hline $\begin{array}{l}\text { Minaret } \\
\text { specification }\end{array}$ & $\mathrm{A}$ & $\mathrm{I}$ & $\mathrm{R}$ & $\mathrm{T}(\mathrm{sec})$ & $\mathrm{B}$ & $\mathrm{C}$ & $\mathrm{W}($ ton & $\mathrm{V}($ ton $)$ & $\begin{array}{l}\mathrm{K}(\text { to } \\
\mathrm{n} / \mathrm{m} \\
\text { ) }\end{array}$ & $\begin{array}{l}\mathrm{U}(\mathrm{cm} \\
\text { ) }\end{array}$ \\
\hline $\begin{array}{l}\text { Hand- operated } \\
\text { method }\end{array}$ & 0.30 & 1.2 & 5 & 0.758 & 2.5 & $\begin{array}{l}0.1 \\
8\end{array}$ & 92.53 & 16.66 & 380 & 4.39 \\
\hline Computer method & 0.30 & 1.2 & 5 & 0.870 & 2.5 & $\begin{array}{l}0.1 \\
8\end{array}$ & 94.3 & 17.33 & 347 & 5 \\
\hline
\end{tabular}

Table 1: Static analysis results

Because of excessive calculations in hand- operated method, the details are not presented. Also, in SAP2000, modeling is presented on a cylindered minaret in height, diameter and thickness equal to $30 \mathrm{~m}, 2 \mathrm{~m}$ and $20 \mathrm{~cm}$, respectively (fig.1).

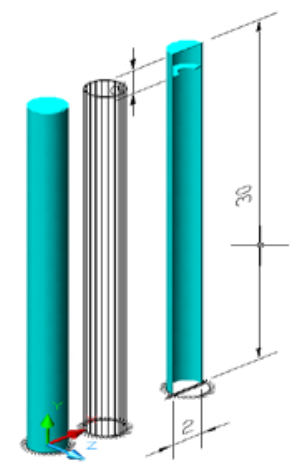

Fig.1: Geometric specification of supposed concrete minaret with fixed support

\section{Data modeling using GP}

150 samples of concrete minaret were modeled using SAP 2000 software in different length, diameter and thickness (inputs) and two parameters include minaret base shear and top lateral displacement (outputs), and transferred to Genetic programing to provide the best model for concrete minaret modeling.

Specifications of 150 concrete minaret samples were used in GP training. Minaret specification includes 7 parameters: length, diameter, thickness, elasticity modulus, poison's ratio, compressive strength and density. Length, diameter and thickness variability in concrete minaret is $20-70 \mathrm{~m}, 2-7 \mathrm{~m}$ and $0.2-0.45 \mathrm{~m}$, respectively. General characteristics of used concrete minaret are provided in table 2.

\begin{tabular}{|l|l|l|l|l|}
\hline $\begin{array}{l}\text { Concrete } \\
\text { specifications }\end{array}$ & $\begin{array}{l}\text { poison's ratio } \\
(v)\end{array}$ & $\begin{array}{l}\text { Elasticity } \\
\text { modulus (E) } \\
\mathrm{kg} / \mathrm{m} /\end{array}$ & $\begin{array}{l}\text { Density }(\gamma) \\
\mathrm{kg} / \mathrm{m3}\end{array}$ & $\begin{array}{l}\text { Compressive } \\
\text { strength }\left(f^{\prime} c\right) \\
\mathrm{kg} / \mathrm{cm}^{2}\end{array}$ \\
\hline $\begin{array}{l}\text { Concrete } \\
\text { minaret }\end{array}$ & 2 & 2188197889 & 2400 & 210 \\
\hline
\end{tabular}

Table 2: General characteristics of used concrete minaret

In GP modeling, suitable GP figuration is needed which is primary setting and determination of mathematic operators. 


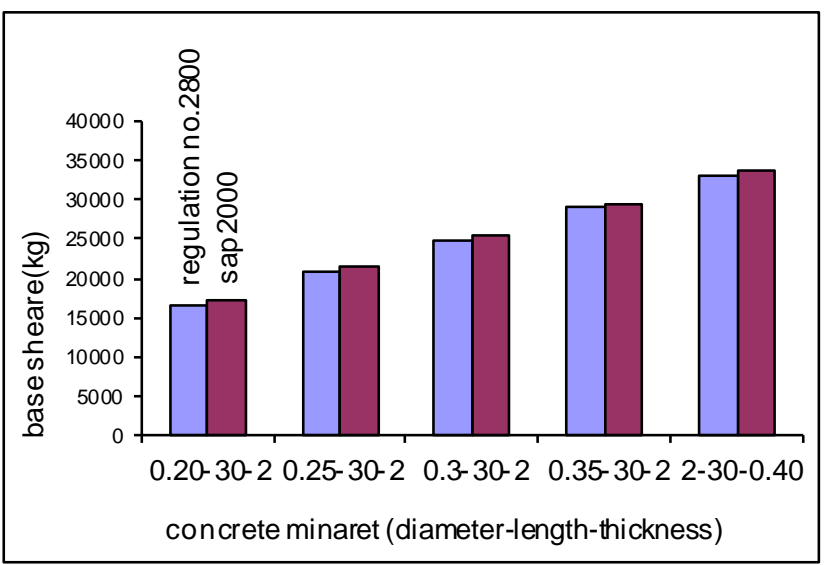

Fig.4: Base shearing on the base of fixed diameter and height but variable thickness

口

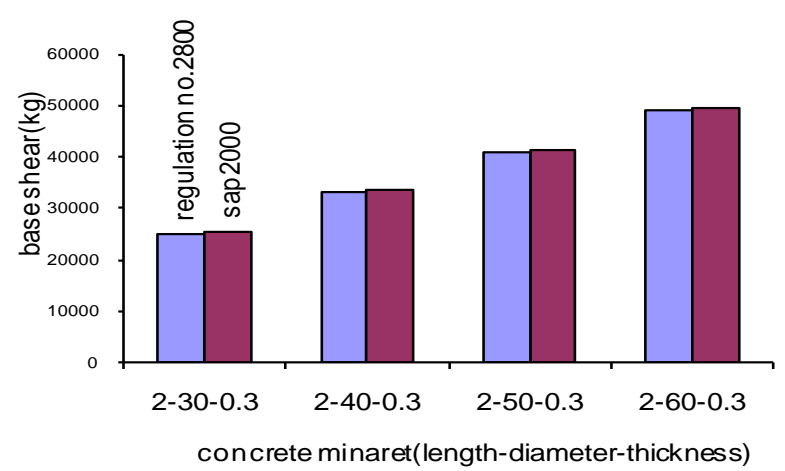

Fig.6: Base shearing on the base of fixed diameter and thickness but variable height thickness but

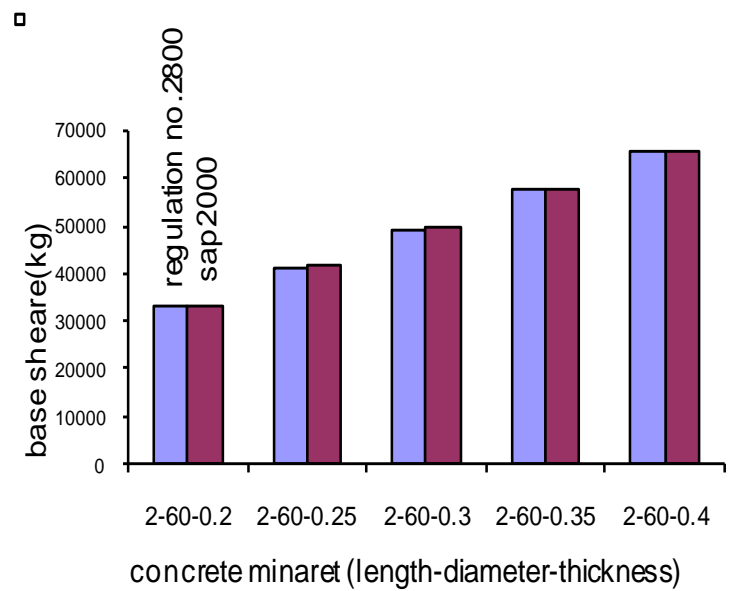

Fig.8: Base shearing on the base of fixed height and thickness but variable diameter

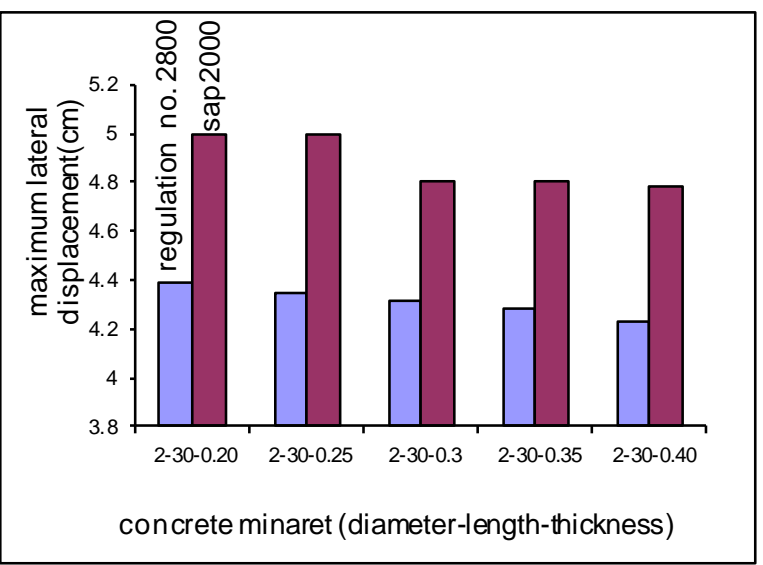

Fig.3: Maximum lateral displacement on the base of fixed diameter and height but variable thickness

口

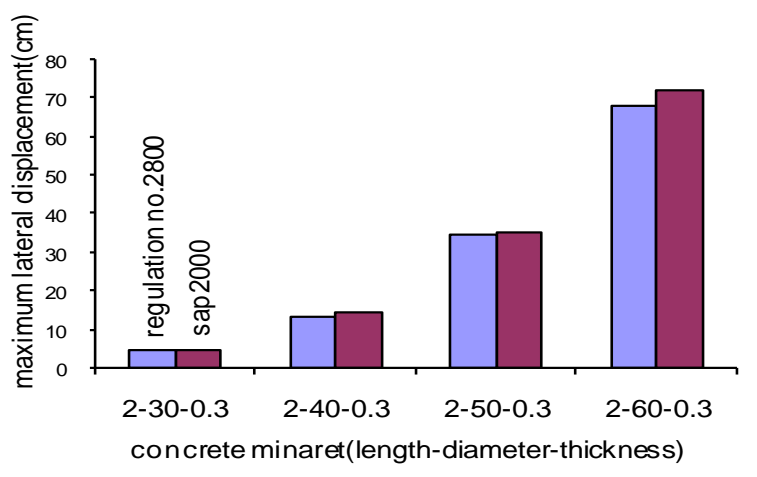

Fig.5: Maximum lateral displacement on the base of fixed diameter and height

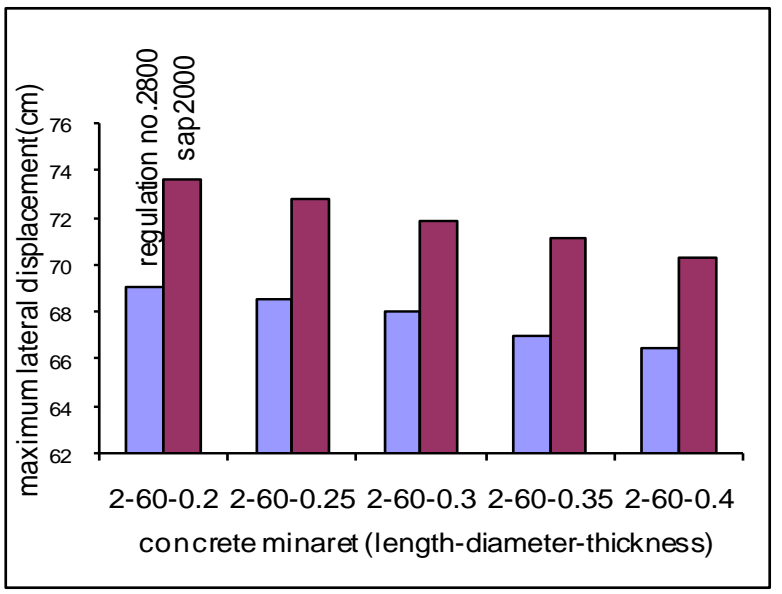

Fig.7: Maximum lateral displacement on the base of fixed height and thickness but variable diameter 


\section{Primary setting}

In adjusting, the follow factors should be considered in genetic programing:

1. Data value and number of variables (table 3)

2. General settings include number of chromosomes and gens, size of head, tail, the place of division and gen linking function (table 4)

3. Fitness function (table 5)

4. Genetic operators (table 6)

5. Numerical constants (table 7)

6.

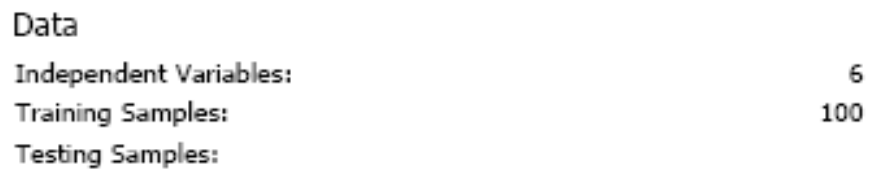

Table 3: data

$\begin{array}{lr}\text { Settings } & \\ \text { General } & \\ \text { Chromosomes: } & 30 \\ \text { Genes: } & 3 \\ \text { Head Size: } & 8 \\ \text { Tail Size: } & 25 \\ \text { Dc Size: } & 25 \\ \text { Gene Size: } & 58 \\ \text { Linking Function: } & \text { Addition }\end{array}$

l'able 4: General settings

Fitness Function

Error Type:

RRSE

Precision:

Selection Range:

Table 5: fitness function

$\begin{array}{lr}\text { Genetic Operators } & \\ \text { Mutation Rate: } & 0.044 \\ \text { Inversion Rate: } & 0.1 \\ \text { IS Transposition Rate: } & 0.1 \\ \text { RIS Transposition Rate: } & 0.1 \\ \text { One-Point Recombination Rate: } & 0.3 \\ \text { Two-Point Recombination Rate: } & 0.3 \\ \text { Gene Recombination Rate: } & 0.1 \\ \text { Gene Transposition Rate: } & 0.1\end{array}$

Table 6: genetic operators 
Kaveh Kumarci, Afsaneh Banitalebi Dehkordi / TJ MCS Vol .4 No.4 (2012) 570-584

$\begin{array}{lr}\text { Numerical Constants } & 2 \\ \text { Constants per Gene: } & \text { Floating-Point } \\ \text { Data Type: } & -10 \\ \text { Lower Bound: } & 10 \\ \text { Upper Bound: } & 0.01 \\ \text { RNC Mutation: } & 0.044 \\ \text { DC Mutation: } & 0.1 \\ \text { Dc Inversion: } & 0.1 \\ \text { DC IS Transposition: } & \end{array}$

Table 7: numerical constant

\section{Top displacement analysis}

\section{SAP 2000 analysis in minaret top displacement}

The analysis of 50 samples of concrete minaret in diameter of $5.5 \mathrm{~m}$ was performed and top displacement output is shown in table 8.

\begin{tabular}{|c|c|c|c|c|c|}
\hline No. & $\begin{array}{l}\text { Average } \\
\text { diameter } \\
\text { (m) }\end{array}$ & $\begin{array}{l}\text { Length } \\
\text { (m) }\end{array}$ & $\begin{array}{l}\text { Thickness } \\
\text { (m) }\end{array}$ & Weight (kg) & $\begin{array}{l}\text { Top displacement } \\
\text { determined using } \\
\text { SAP } 2000\end{array}$ \\
\hline 1 & 5.5 & 21 & 0.21 & 199686.4267 & 0.001796962 \\
\hline 2 & 5.5 & 21 & 0.26 & 243531.5863 & 0.001746532 \\
\hline 3 & 5.5 & 21 & 0.31 & 287379.3848 & 0.001711733 \\
\hline 4 & 5.5 & 21 & 0.36 & 331229.8223 & 0.001685949 \\
\hline 5 & 5.5 & 21 & 0.41 & 375082.8987 & 0.001665793 \\
\hline 6 & 5.5 & 26 & 0.21 & 243228.9001 & 0.004130076 \\
\hline 7 & 5.5 & 26 & 0.26 & 297441.3153 & 0.004028043 \\
\hline 8 & 5.5 & 26 & 0.31 & 351656.3694 & 0.00395743 \\
\hline 9 & 5.5 & 26 & 0.36 & 405874.0625 & 0.003904905 \\
\hline 10 & 5.5 & 26 & 0.41 & 460094.3945 & 0.003863639 \\
\hline 11 & 5.5 & 31 & 0.21 & 286771.3736 & 0.008203811 \\
\hline 12 & 5.5 & 31 & 0.26 & 351351.0443 & 0.008023087 \\
\hline 13 & 5.5 & 31 & 0.31 & 415933.354 & 0.007897648 \\
\hline 14 & 5.5 & 31 & 0.36 & 480518.3027 & 0.007803971 \\
\hline 15 & 5.5 & 31 & 0.41 & 545105.8902 & 0.007730008 \\
\hline 16 & 5.5 & 36 & 0.21 & 330313.847 & 0.014716984 \\
\hline 17 & 5.5 & 36 & 0.26 & 405260.7734 & 0.014424578 \\
\hline 18 & 5.5 & 36 & 0.31 & 480210.3386 & 0.014221025 \\
\hline 19 & 5.5 & 36 & 0.36 & 555162.5429 & 0.014068412 \\
\hline
\end{tabular}


Kaveh Kumarci, Afsaneh Banitalebi Dehkordi / TJ MCS Vol .4 No.4 (2012) 570-584

\begin{tabular}{|c|c|c|c|c|c|}
\hline 20 & 5.5 & 36 & 0.41 & 630117.386 & 0.01394733 \\
\hline 21 & 5.5 & 41 & 0.21 & 373856.3205 & 0.024485716 \\
\hline 22 & 5.5 & 41 & 0.26 & 459170.5024 & 0.024042645 \\
\hline 23 & 5.5 & 41 & 0.31 & 544487.3232 & 0.0237333 \\
\hline 24 & 5.5 & 41 & 0.36 & 629806.783 & 0.023500468 \\
\hline 25 & 5.5 & 41 & 0.41 & 715128.8818 & 0.023314856 \\
\hline 26 & 5.5 & 46 & 0.21 & 417398.7939 & 0.038443435 \\
\hline 27 & 5.5 & 46 & 0.26 & 513080.2314 & 0.037804627 \\
\hline 28 & 5.5 & 46 & 0.31 & 608764.3078 & 0.037357318 \\
\hline 29 & 5.5 & 46 & 0.36 & 704451.0232 & 0.037019351 \\
\hline 30 & 5.5 & 46 & 0.41 & 800140.3775 & 0.036748666 \\
\hline 31 & 5.5 & 51 & 0.21 & 460941.2673 & 0.057640868 \\
\hline 32 & 5.5 & 51 & 0.26 & 566989.9604 & 0.056755076 \\
\hline 33 & 5.5 & 51 & 0.31 & 673041.2924 & 0.056133025 \\
\hline 34 & 5.5 & 51 & 0.36 & 779095.2634 & 0.055661249 \\
\hline 35 & 5.5 & 51 & 0.41 & 885151.8733 & 0.055281663 \\
\hline 36 & 5.5 & 56 & 0.21 & 504483.7408 & 0.083246049 \\
\hline 37 & 5.5 & 56 & 0.26 & 620899.6894 & 0.082055759 \\
\hline 38 & 5.5 & 56 & 0.31 & 737318.277 & 0.081217469 \\
\hline 39 & 5.5 & 56 & 0.36 & 853739.5036 & 0.080579321 \\
\hline 40 & 5.5 & 56 & 0.41 & 970163.369 & 0.080063578 \\
\hline 41 & 5.5 & 61 & 0.21 & 548026.2142 & 0.116544315 \\
\hline 42 & 5.5 & 61 & 0.26 & 674809.4184 & 0.114985653 \\
\hline 43 & 5.5 & 61 & 0.31 & 801595.2616 & 0.113884802 \\
\hline 44 & 5.5 & 61 & 0.36 & 928383.7437 & 0.1130437 \\
\hline 45 & 5.5 & 61 & 0.41 & 1055174.865 & 0.112360965 \\
\hline 46 & 5.5 & 66 & 0.21 & 591568.6876 & 0.158938305 \\
\hline 47 & 5.5 & 66 & 0.26 & 728719.1475 & 0.15694095 \\
\hline 48 & 5.5 & 66 & 0.31 & 865872.2462 & 0.155526278 \\
\hline 49 & 5.5 & 66 & 0.36 & 1003027.984 & 0.154441494 \\
\hline 50 & 5.5 & 66 & 0.41 & 1140186.361 & 0.153557206 \\
\hline
\end{tabular}

Table 8: The results of concrete minaret analysis with top displacement output using SAP 2000 
GP modeling in minaret top displacement: Using data in table 8 as primary population in genetic programing, some models were provided. Specifications the best model is shown in figure 9 and mathematical details are provided in figure 10.

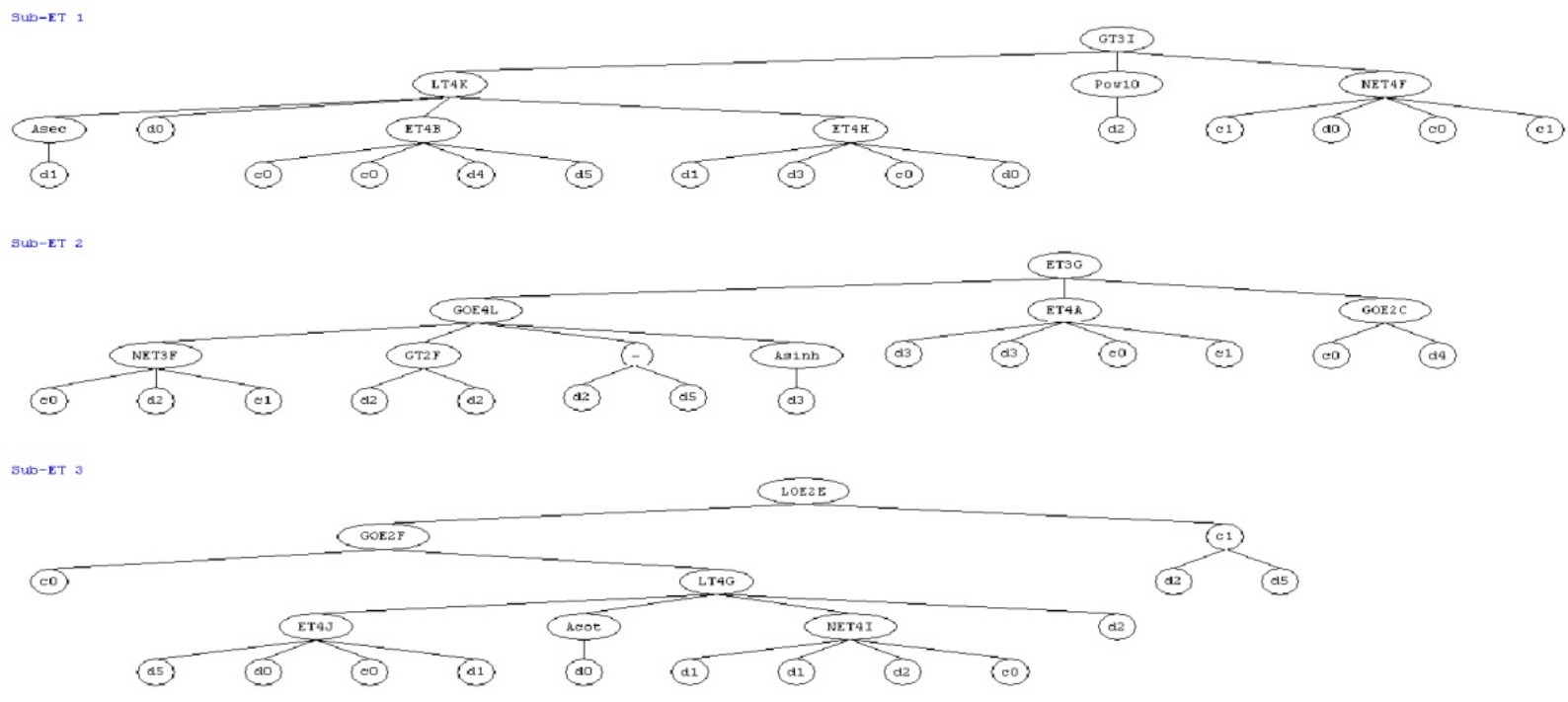

Fig.9: Genetic programing tree in modeling of base shear calculation in concrete minaret in diameter of $5.5 \mathrm{~m}$

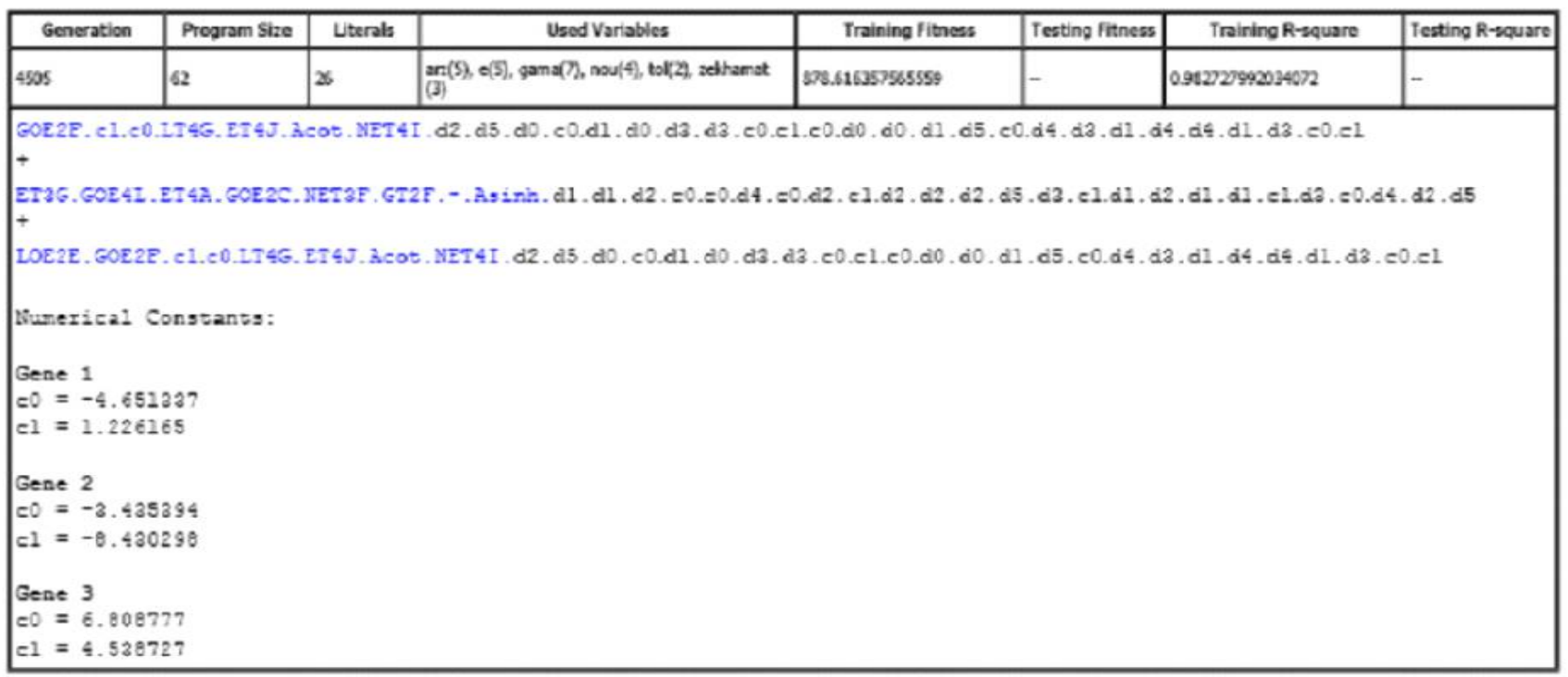

Figure 10. Mathematic formula details of the best genetic programming model in calculation of base shear in concrete minaret with diameter of $5.5 \mathrm{~m}$

\section{Test of model}

\section{Comparing concrete minaret top displacement in SAP2000 and Genetic Programing}

To test of models, secondary population is needed, so that non of them don't find in initial population. Then we compare the results of minaret top displacement and base shearing in SAP2000 and Genetic Programing. Once again, 50 minarets were chose to compare the results of SAP 2000 and GP analysis. The results are presented in table 10. Also, figure 11 implies comparison diagram of error percent. In this model, the average of error percent is $12.5 \%$. 
Kaveh Kumarci, Afsaneh Banitalebi Dehkordi / TJ MCS Vol .4 No.4 (2012) 570-584

\begin{tabular}{|c|c|c|c|c|c|c|c|}
\hline No. & $\begin{array}{r}\text { Diameter } \\
(\mathrm{m})\end{array}$ & $\begin{array}{r}\text { Height } \\
(\mathrm{m})\end{array}$ & $\begin{array}{r}\text { Thickness } \\
(\mathrm{m})\end{array}$ & Weight (kg) & $\begin{array}{r}\text { Top } \\
\text { displacement } \\
\text { using } \\
\text { SAP2000 (m) }\end{array}$ & $\begin{array}{r}\text { Top } \\
\text { displacement } \\
\text { using GP (m) }\end{array}$ & $\begin{array}{r}\text { Error } \\
\text { percent }\end{array}$ \\
\hline 1 & 6 & 20 & 0.2 & 200843.8874 & 0.001276468 & 0.001222916 & -4.19529263 \\
\hline 2 & 6 & 20 & 0.25 & 246411.3686 & 0.001233724 & 0.001125334 & $\begin{array}{l}- \\
8.785572297\end{array}$ \\
\hline 3 & 6 & 20 & 0.3 & 291981.4888 & 0.001204861 & 0.00120286 & $\begin{array}{l}- \\
0.166043496\end{array}$ \\
\hline 4 & 6 & 20 & 0.35 & 337554.2478 & 0.001183866 & 0.001311585 & 10.78829956 \\
\hline 5 & 6 & 20 & 0.4 & 383129.6458 & 0.001167736 & 0.001145231 & $\begin{array}{l}- \\
1.927194554\end{array}$ \\
\hline 6 & 6 & 25 & 0.2 & 246082.8209 & 0.003036567 & 0.003064911 & 0.9334243 \\
\hline 7 & 6 & 25 & 0.25 & 302960.0354 & 0.00294716 & 0.002891449 & $\begin{array}{l}- \\
1.890328889\end{array}$ \\
\hline 8 & 6 & 25 & 0.3 & 359839.8889 & 0.002886659 & 0.003076267 & 6.568404466 \\
\hline 9 & 6 & 25 & 0.35 & 416722.3814 & 0.002842524 & 0.002834937 & $\begin{array}{l}- \\
0.266906168\end{array}$ \\
\hline 10 & 6 & 25 & 0.4 & 473607.5127 & 0.002808486 & 0.003059307 & 8.930841214 \\
\hline 11 & 6 & 30 & 0.2 & 291321.7543 & 0.006170338 & 0.005724264 & $\begin{array}{l}- \\
7.229331397\end{array}$ \\
\hline 12 & 6 & 30 & 0.25 & 359508.7022 & 0.006008597 & 0.006608136 & 9.978015297 \\
\hline 13 & 6 & 30 & 0.3 & 427698.2891 & 0.005898913 & 0.005499492 & $\begin{array}{l}- \\
6.771104434\end{array}$ \\
\hline 14 & 6 & 30 & 0.35 & 495890.5149 & 0.005818662 & 0.005508171 & $\begin{array}{l}- \\
5.336118473 \\
\end{array}$ \\
\hline 15 & 6 & 30 & 0.4 & 564085.3796 & 0.005756534 & 0.005655093 & $\begin{array}{l}- \\
1.762185524\end{array}$ \\
\hline 16 & 6 & 35 & 0.2 & 336560.6878 & 0.011248991 & 0.011582485 & 2.964660508 \\
\hline 17 & 6 & 35 & 0.25 & 416057.369 & 0.010983494 & 0.010258071 & - 6.604661719 \\
\hline 18 & 6 & 35 & 0.3 & 495556.6892 & 0.010803059 & 0.012239889 & 13.30022269 \\
\hline 19 & 6 & 35 & 0.35 & 575058.6484 & 0.01067065 & 0.011166848 & 4.65012082 \\
\hline 20 & 6 & 35 & 0.4 & 654563.2465 & 0.010567754 & 0.009968588 & $\begin{array}{l}- \\
5.669765782\end{array}$ \\
\hline 21 & 6 & 40 & 0.2 & 381799.6212 & 0.018942335 & 0.021477294 & 13.38250381 \\
\hline 22 & 6 & 40 & 0.25 & 472606.0358 & 0.018535847 & 0.019952082 & 7.640520487 \\
\hline 23 & 6 & 40 & 0.3 & 563415.0894 & 0.018258996 & 0.020648842 & 13.08859269 \\
\hline 24 & 6 & 40 & 0.35 & 654226.7819 & 0.018055236 & 0.01890349 & 4.698106833 \\
\hline 25 & 6 & 40 & 0.4 & 745041.1134 & 0.017896297 & 0.018183567 & 1.605188477 \\
\hline 26 & 6 & 45 & 0.2 & 427038.5546 & 0.030018783 & 0.031881031 & 6.203609183 \\
\hline 27 & 6 & 45 & 0.25 & 529154.7026 & 0.029428195 & 0.031195212 & 6.004506335 \\
\hline
\end{tabular}


Kaveh Kumarci, Afsaneh Banitalebi Dehkordi / TJ MCS Vol .4 No.4 (2012) 570-584

\begin{tabular}{|c|c|c|c|c|c|c|c|}
\hline 28 & 6 & 45 & 0.3 & 631273.4896 & 0.029025093 & 0.029751944 & 2.504216615 \\
\hline 29 & 6 & 45 & 0.35 & 733394.9154 & 0.028727542 & 0.030404063 & 5.835936688 \\
\hline 30 & 6 & 45 & 0.4 & 835518.9802 & 0.028494586 & 0.026660707 & 6.435884571 \\
\hline 31 & 6 & 50 & 0.2 & 472277.4881 & 0.045345348 & 0.04881875 & 7.659887248 \\
\hline 32 & 6 & 50 & 0.25 & 585703.3694 & 0.044521616 & 0.04616313 & 3.687002988 \\
\hline 33 & 6 & 50 & 0.3 & 699131.8897 & 0.043958179 & 0.049028885 & 11.53529686 \\
\hline 34 & 6 & 50 & 0.35 & 812563.049 & 0.043541069 & 0.048857337 & 12.20977993 \\
\hline 35 & 6 & 50 & 0.4 & 925996.8471 & 0.043213319 & 0.049397223 & 14.31018177 \\
\hline 36 & 6 & 55 & 0.2 & 517516.4215 & 0.065887646 & 0.060520557 & -8.14581923 \\
\hline 37 & 6 & 55 & 0.25 & 642252.0362 & 0.064775731 & 0.069369308 & 7.091510165 \\
\hline 38 & 6 & 55 & 0.3 & 766990.2899 & 0.064013552 & 0.071880364 & 12.28929149 \\
\hline 39 & 6 & 55 & 0.35 & 891731.1825 & 0.063447692 & 0.064646453 & 1.889367605 \\
\hline 40 & 6 & 55 & 0.4 & 1016474.714 & 0.063001469 & 0.065728635 & 4.328733922 \\
\hline 41 & 6 & 60 & 0.2 & 562755.355 & 0.092709894 & 0.101146595 & 9.100107854 \\
\hline 42 & 6 & 60 & 0.25 & 698800.703 & 0.091248696 & 0.098811402 & 8.288015535 \\
\hline 43 & 6 & 60 & 0.3 & 834848.69 & 0.090244973 & 0.086656375 & $\begin{array}{l}- \\
3.976507763\end{array}$ \\
\hline 44 & 6 & 60 & 0.35 & 970899.316 & 0.089497667 & 0.09655449 & 7.88492467 \\
\hline 45 & 6 & 60 & 0.4 & 1106952.581 & 0.088906284 & 0.091678905 & 3.118587661 \\
\hline 46 & 6 & 65 & 0.2 & 607994.2884 & 0.126974913 & 0.121623575 & - \\
\hline 47 & 6 & 65 & 0.25 & 755349.3698 & 0.125097213 & 0.135000775 & 7.916693226 \\
\hline 48 & 6 & 65 & 0.3 & 902707.0902 & 0.12380467 & 0.12891223 & 4.125498529 \\
\hline 49 & 6 & 65 & 0.35 & 1050067.45 & 0.122839622 & 0.133149748 & 8.393160706 \\
\hline 50 & 6 & 65 & 0.4 & 1197430.448 & 0.122073286 & 0.113633113 & 6.914021778 \\
\hline
\end{tabular}

Table 10:Comparing the best model of SAP2000 and GP for minaret top displacement in diameter of $6 \mathrm{~m}$ 


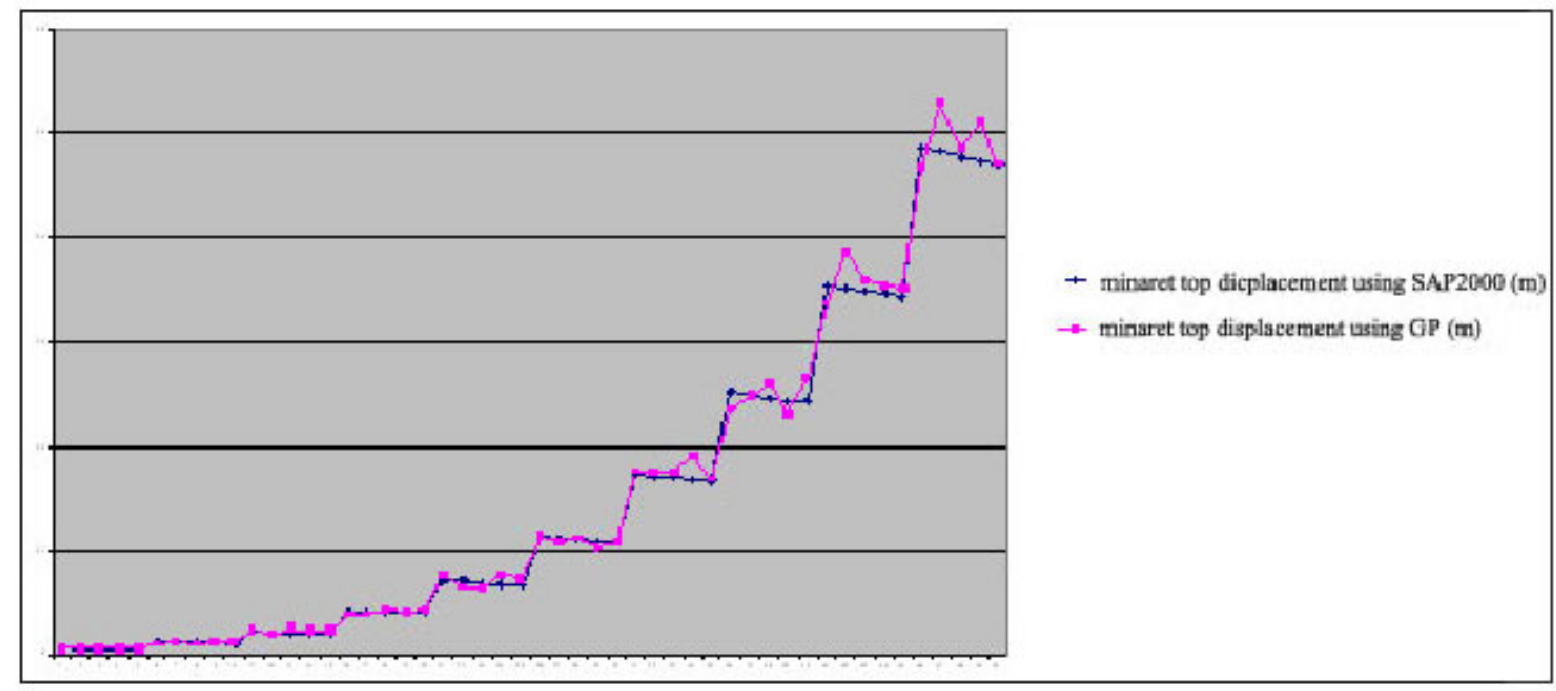

Fig.11: Comparing the best model of genetic programming and SAP2000 software in top displacement of concrete minaret with diameter of $6 \mathrm{~m}$.

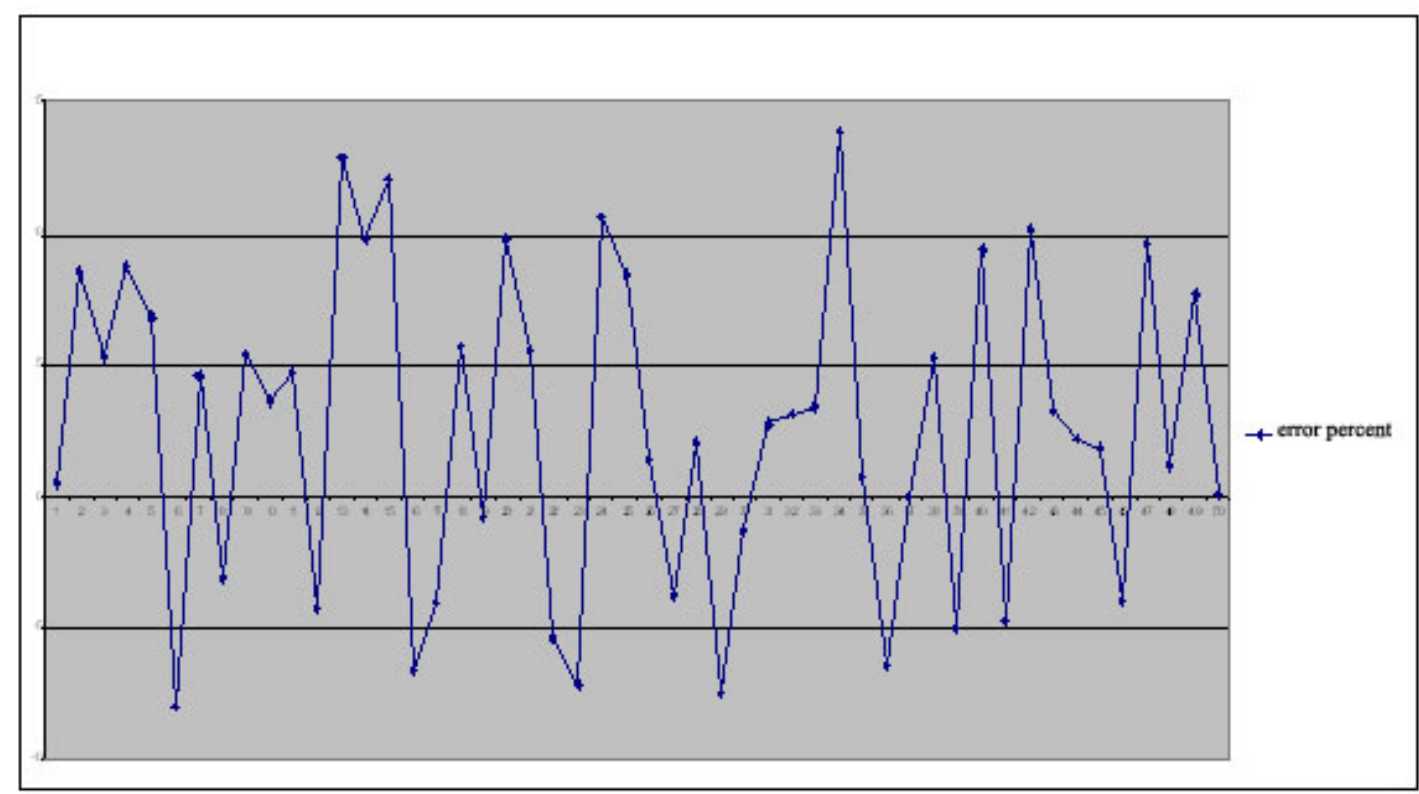

Figure 12. Comparing error percent of the best the best model of genetic programming and SAP2000 software in calculation of top displacement of concrete minaret with diameter of $6 \mathrm{~m}$

\section{Comparing concrete minaret base shearing in SAP 2000 and Genetic Programing}

Results of base shearing calculated in GP and SAP 2000 are provided in table 11. Also, the results and error percent in samples are depicted in figures 13 and 14, respectively. The average of error percent in this model is $12.5 \%$.

\begin{tabular}{|l|l|l|l|l|l|l|l|}
\hline No. & $\begin{array}{r}\text { Diameter } \\
(\mathrm{m})\end{array}$ & $\begin{array}{r}\text { Height } \\
(\mathrm{m})\end{array}$ & $\begin{array}{r}\text { Thickness } \\
(\mathrm{m})\end{array}$ & Weight $(\mathrm{kg})$ & $\begin{array}{r}\text { Base } \\
\text { using SAP2000 } \\
(\mathrm{kg})\end{array}$ & $\begin{array}{r}\text { Base shear } \\
\text { using GP (kg) }\end{array}$ & Error percent \\
\hline 1 & 6 & 20 & 0.2 & 200843.8874 & 36151.89974 & 35197.30821 & -2.640501716 \\
\hline 2 & 6 & 20 & 0.25 & 246411.3686 & 44354.04635 & 40878.21729 & -7.836554606 \\
\hline
\end{tabular}


Kaveh Kumarci, Afsaneh Banitalebi Dehkordi / TJ MCS Vol .4 No.4 (2012) 570-584

\begin{tabular}{|c|c|c|c|c|c|c|c|}
\hline 3 & 6 & 20 & 0.3 & 291981.4888 & 52556.66798 & 61082.79476 & 16.22273084 \\
\hline 4 & 6 & 20 & 0.35 & 337554.2478 & 60759.76461 & 67267.44603 & 10.71051123 \\
\hline 5 & 6 & 20 & 0.4 & 383129.6458 & 68963.33625 & 75147.79082 & 8.967742719 \\
\hline 6 & 6 & 25 & 0.2 & 246082.8209 & 44294.90776 & 42164.99611 & -4.808479693 \\
\hline 7 & 6 & 25 & 0.25 & 302960.0354 & 54532.80638 & 63750.34633 & 16.9027427 \\
\hline 8 & 6 & 25 & 0.3 & 359839.8889 & 64771.18001 & 62275.96488 & -3.852353978 \\
\hline 9 & 6 & 25 & 0.35 & 416722.3814 & 75010.02864 & 72416.98333 & -3.456931503 \\
\hline 10 & 6 & 25 & 0.4 & 473607.5127 & 85249.35229 & 94436.57678 & 10.77688479 \\
\hline 11 & 6 & 30 & 0.2 & 291321.7543 & 52437.91578 & 56177.97709 & 7.132360725 \\
\hline 12 & 6 & 30 & 0.25 & 359508.7022 & 64711.5664 & 58264.59691 & -9.962623144 \\
\hline 13 & 6 & 30 & 0.3 & 427698.2891 & 76985.69204 & 68927.96338 & -10.46652754 \\
\hline 14 & 6 & 30 & 0.35 & 495890.5149 & 89260.29268 & 103302.7054 & 15.73198146 \\
\hline 15 & 6 & 30 & 0.4 & 564085.3796 & 101535.3683 & 110985.8779 & 9.307603615 \\
\hline 16 & 6 & 35 & 0.2 & 336560.6878 & 60580.9238 & 54757.70107 & -9.612304275 \\
\hline 17 & 6 & 35 & 0.25 & 416057.369 & 74890.32643 & 70773.1551 & -5.497600991 \\
\hline 18 & 6 & 35 & 0.3 & 495556.6892 & 89200.20406 & 87119.16129 & -2.333002259 \\
\hline 19 & 6 & 35 & 0.35 & 575058.6484 & 103510.5567 & 104177.4374 & 0.644263434 \\
\hline 20 & 6 & 35 & 0.4 & 654563.2465 & 117821.3844 & 120217.4555 & 2.033647105 \\
\hline 21 & 6 & 40 & 0.2 & 381799.6212 & 68723.93182 & 67498.67727 & -1.782864447 \\
\hline 22 & 6 & 40 & 0.25 & 472606.0358 & 85069.08645 & 77901.07093 & -8.426110842 \\
\hline 23 & 6 & 40 & 0.3 & 563415.0894 & 101414.7161 & 116699.9515 & 15.07200928 \\
\hline 24 & 6 & 40 & 0.35 & 654226.7819 & 117760.8207 & 121055.9248 & 2.798132693 \\
\hline 25 & 6 & 40 & 0.4 & 745041.1134 & 134107.4004 & 129853.8854 & -3.171722823 \\
\hline 26 & 6 & 45 & 0.2 & 427038.5546 & 76866.93984 & 75683.59205 & -1.539475606 \\
\hline 27 & 6 & 45 & 0.25 & 529154.7026 & 95247.84647 & 90393.90054 & -5.096121451 \\
\hline 28 & 6 & 45 & 0.3 & 631273.4896 & 113629.2281 & 129405.5961 & 13.88407563 \\
\hline 29 & 6 & 45 & 0.35 & 733394.9154 & 132011.0848 & 128231.7611 & -2.862883625 \\
\hline 30 & 6 & 45 & 0.4 & 835518.9802 & 150393.4164 & 143658.2176 & -4.478386734 \\
\hline 31 & 6 & 50 & 0.2 & 472277.4881 & 85009.94786 & 82852.82084 & -2.537499519 \\
\hline 32 & 6 & 50 & 0.25 & 585703.3694 & 105426.6065 & 118505.044 & 12.40525319 \\
\hline 33 & 6 & 50 & 0.3 & 699131.8897 & 125843.7402 & 127633.4051 & 1.422132683 \\
\hline 34 & 6 & 50 & 0.35 & 812563.049 & 146261.3488 & 135141.7985 & -7.602521385 \\
\hline 35 & 6 & 50 & 0.4 & 925996.8471 & 166679.4325 & 190880.9408 & 14.51979282 \\
\hline
\end{tabular}


Kaveh Kumarci, Afsaneh Banitalebi Dehkordi / TJ MCS Vol .4 No.4 (2012) 570-584

\begin{tabular}{|l|l|l|l|l|l|l|l|}
\hline 36 & 6 & 55 & 0.2 & 517516.4215 & 93152.95587 & 93450.25128 & 0.319147584 \\
\hline 37 & 6 & 55 & 0.25 & 642252.0362 & 115605.3665 & 126549.7946 & 9.467058857 \\
\hline 38 & 6 & 55 & 0.3 & 766990.2899 & 138058.2522 & 155058.0241 & 12.3134776 \\
\hline 39 & 6 & 55 & 0.35 & 891731.1825 & 160511.6128 & 185852.563 & 15.78761171 \\
\hline 40 & 6 & 55 & 0.4 & 1016474.714 & 182965.4485 & 197055.804 & 7.701101805 \\
\hline 41 & 6 & 60 & 0.2 & 562755.355 & 101295.9639 & 95710.52152 & -5.513983143 \\
\hline 42 & 6 & 60 & 0.25 & 698800.703 & 125784.1265 & 130895.9246 & 4.063945291 \\
\hline 43 & 6 & 60 & 0.3 & 834848.69 & 150272.7642 & 165371.6176 & 10.04763136 \\
\hline 44 & 6 & 60 & 0.35 & 970899.316 & 174761.8769 & 191058.185 & 9.324864426 \\
\hline 45 & 6 & 60 & 0.4 & 1106952.581 & 199251.4646 & 221331.5167 & 11.08150054 \\
\hline 46 & 6 & 65 & 0.2 & 607994.2884 & 109438.9719 & 124331.0477 & 13.60765326 \\
\hline 47 & 6 & 65 & 0.25 & 755349.3698 & 135962.8866 & 127720.6701 & -6.062107597 \\
\hline 48 & 6 & 65 & 0.3 & 902707.0902 & 162487.2762 & 156432.4099 & -3.726363349 \\
\hline 49 & 6 & 65 & 0.35 & 1050067.45 & 189012.1409 & 184680.9267 & -2.291500505 \\
\hline 50 & 6 & 65 & 0.4 & 1197430.448 & 215537.4806 & 200367.6932 & -7.038120425 \\
\hline
\end{tabular}

Table 11: Comparing base shear calculated in SAP2000 and GP for minaret in diameter of $6 \mathrm{~m}$

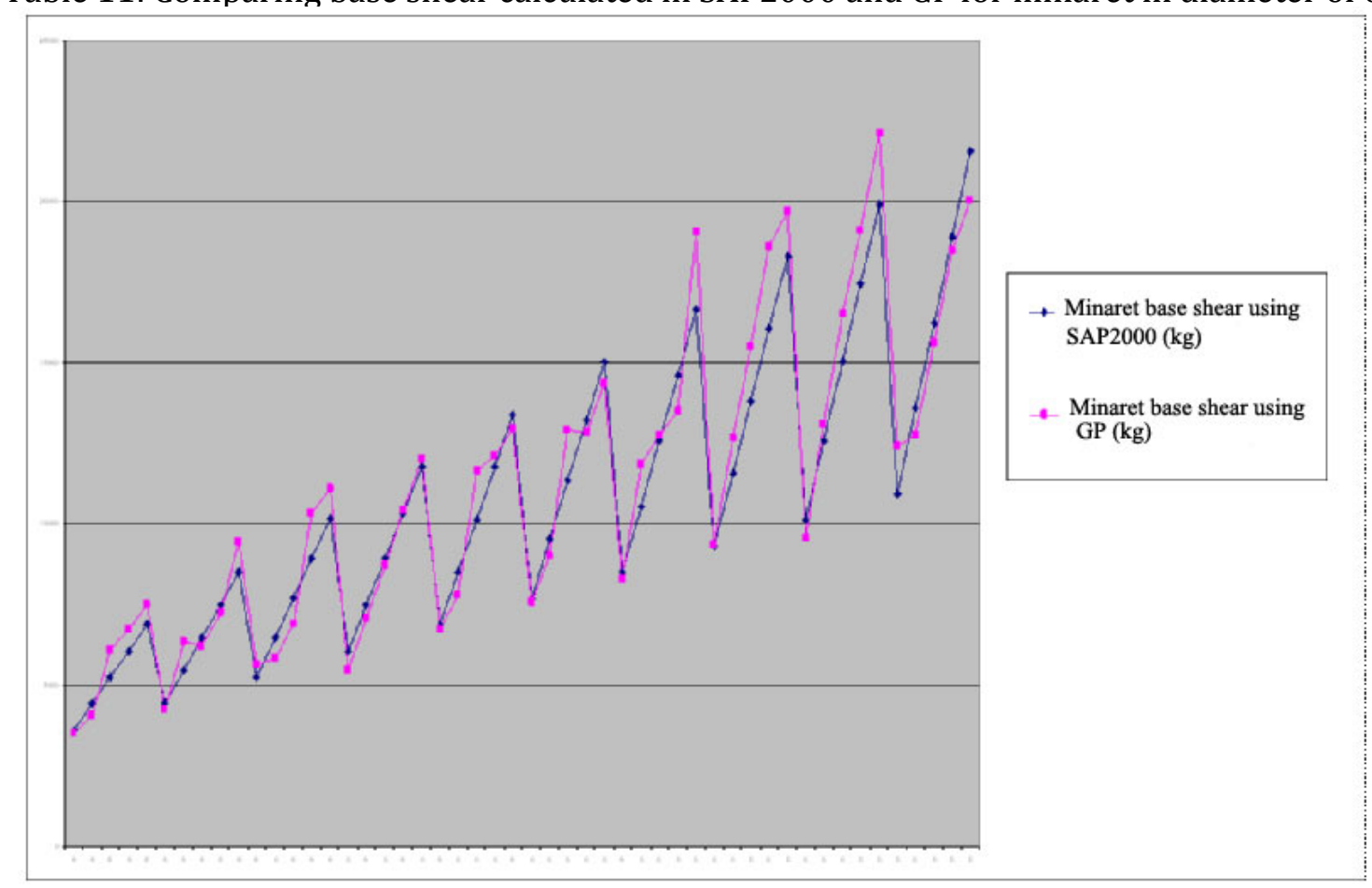

Fig.13: Comparing base shear in GP and SAP2000 for concrete minaret in diameter of $6 \mathrm{~m}$ 


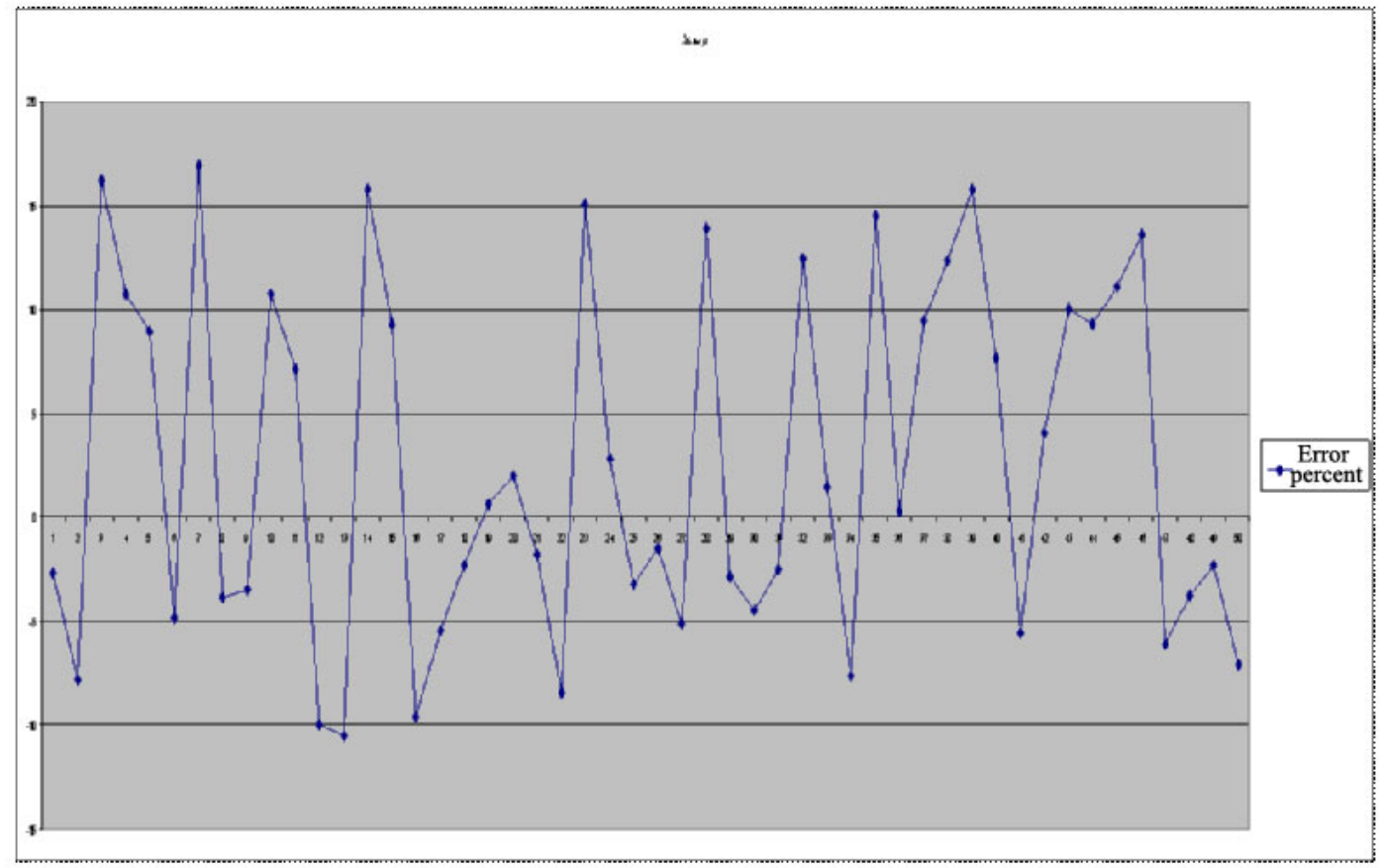

Fig.14: Comparing base shear error percent in GP and SAP2000 for concrete minaret in diameter of $6 \mathrm{~m}$

\section{Conclusions}

Regarding to hand- operated and computer analysis on concrete minarets, it is concluded that in concrete minarets, increase of height leads to increase of base shar and top displacement. Increase of the diameter has increased the base shear and has decreased minaret top displacement. It should be noted that increasing the diameter (above $5 \mathrm{~m}$ ) had no dramatic effect on top displacement decreasing, but base shear increases. Because of increase of structural mass, thickness increasing leads to increase of base shear in acceptable limit, but top displacement is not decrease considerably. According to hand- operated and computer analysis, the following are suggested in design of pendulum structures:

1. Decrease of concrete bulk decreases mass and inertia force.

2. Increase of minaret flexibility leads to increasing natural frequency and decreasing earthquake force,

3. To recognize of structure behavior, using dynamic analysis in pendulum structure is suggested.

Also, we can concluded that according to comparison between error percent in GP models and SAP2000, the results of genetic programing is more acceptable, so GP models can used in maximum lateral top displacement and base shear in concrete minarets.

\section{References}

[1] A.C. Altunisik, "Dynamic response of masonry minarets strengthened with fiber reinforced polymer (FRP) composites", National hazards and earth system sciences, (2011), 20112019.

[2] M. Baei, M. Ghassemieh, A., Goudarzi, "Numerical modeling of end- plate moment connection subjected to bending and axial forces", The Journal Mathematics and Computer Science, Vol.4, No.3, (2012) 463-472. 
[3] A. Dogangun, H. Sezen O. Tuluk, R., Livaoglu R. Acar, "Traditional Turkish monumental structures and their earthquake response", International journal of architectural Heritage, (2007),3(1):251-71.

[4] A.G. El- Attar A.M. Saleh, A.Osman, "Seismic Response of a historical Mamluk style minaret", In: Earthquake resistant engineering structures III, WIT:(2001), pp.745-54.

[5] D.E. Goldberg, "Genetic Algorithms in Search, Optimization and Machine Learning", (1989),Addison-Wesley.

[6] J.R. Koza, genetic programing, Cambridge, (1992), MA:MIT Press/Bradford Books.

[7] M. Mashinchi Joubari, R. Asghari, M. Zareian Jahromy, " Investigation of the dynamic behavior of periodic systems with Newton harmonic balance method", the journal of computer and mathematic science, (2012) Vol.4, No. 3, 418-427.

[8] A. Menon C.G. Lai, G. Macchi "Seismic hazard assessment of historical site of Jam in Afghanistan and stability analysis of minaret", Journal of earthquake engineering, (2004) 8(1): 251-93.

[9] M. Mitchell, "An Introduction to Genetic Algorithms, Series in Complex Adaptive Systems", (1196), Bradford Books/MIT Press.

[10] Montana, D.J., "Strongly typed genetic programing" , (2002), Cambridge, MA 02138.

[11] Q.N. Nguyen,X.H. Neguyen, M. O'Neill, "Semantic aware crossover for genetic programing: the case for real- valued function regression" , EuroGP (2009), LNCS 5481, pp.292-302.

[12] H. Sezen, R. Acar, A. Dogangun, R. Livaoglu, "Dynamic analysis and seismic performance of reinforced concrete minarets, engineering structures", 30(2008) 2253-2264.

[13] C.A. Syrmakezis, "Seismic protection of historical structures and monuments", Structural Control and Health Monitoring, (2006) 13:958-79.

[14] A. Ziaie M.B. Rahnama, :Calculation of Concrete Minaret Frequency by Neural Network", Journal of environmental science and technology, 2(1) (2009), 48-55. 
